\title{
Soteria Bern: ein Schrittmacher in der Schizophreniebehandlung
}

\section{Holger Hoffmann ${ }^{a}$, Sabine Leisinger ${ }^{b}$ \\ a Chefarzt Soteria Bern und Leiter der Einheit für Versorgungsfor- schung an den Universitären Psychiatrischen Diensten Bern \\ b Leiterin Soteria Bern}

Résumé voir page suivante
1 Soteria steht für griechisch Geborgenheit.

Korrespondenz: PD Dr. med. Holger Hoffmann Chefarzt Soteria Bern Bühlstrasse 19A CH-3012 Bern

hoffmann@spk.unibe.ch

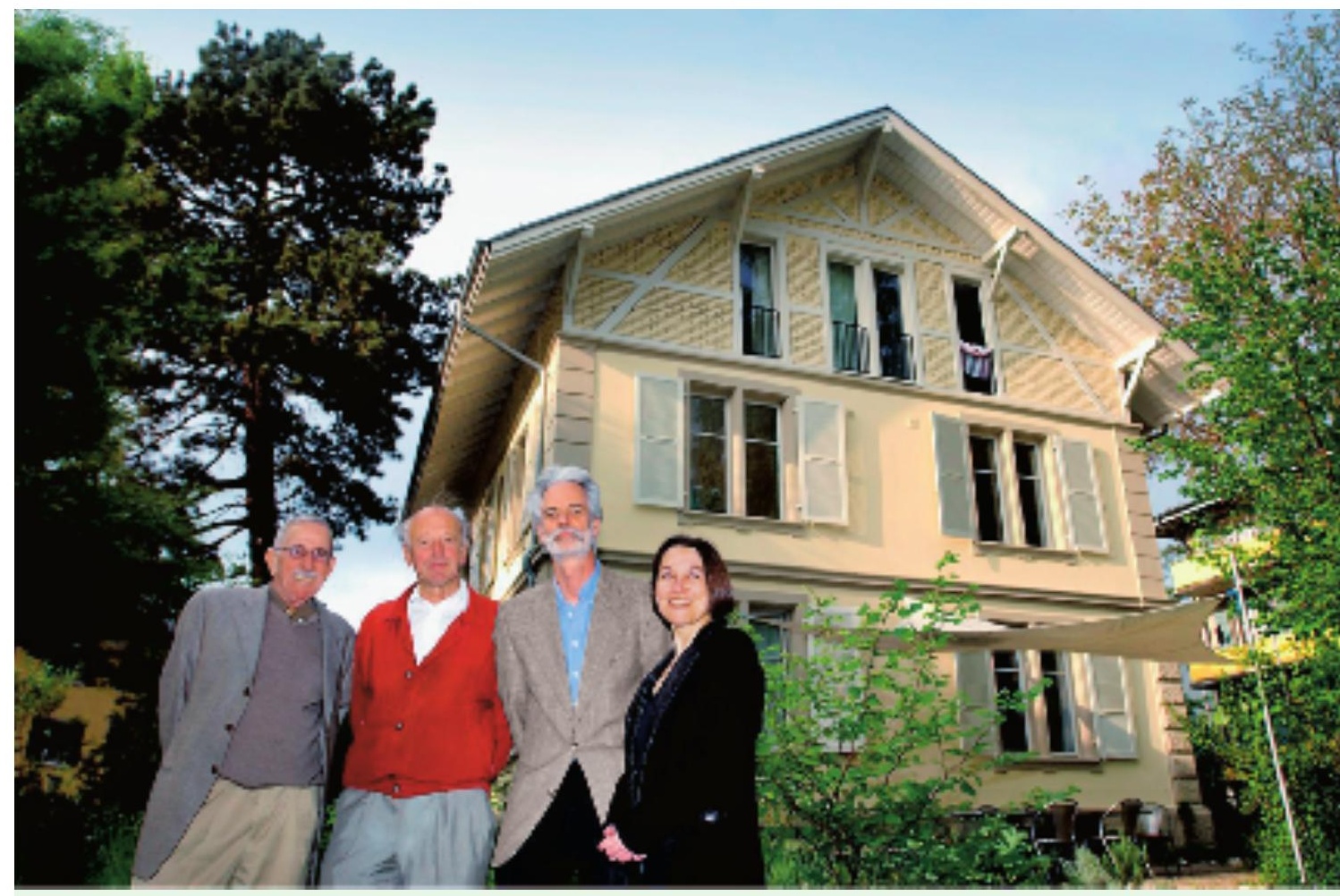

Abbildung 1

Loren Mosher (links), Luc Ciompi und die beiden Autoren vor der Soteria Bern.

\section{Einleitung}

Soteria gilt in weiten Kreisen der Öffentlichkeit und Fachöffentlichkeit als ein Symbol für eine «menschlichere», zugewandte und therapeutisch engagierte Psychiatrie. Standort ausserhalb der Klinik, intensive Milieutherapie, niedrigdosierter Einsatz von Neuroleptika und Einbezug der Angehörigen kennzeichnen das therapeutische Angebot. Die Forschungsresultate aus den Vereinigten Staaten und der Schweiz haben gezeigt, dass der therapeutische Ansatz von Soteria mindestens dieselbe Effektivität bei besserer Effizienz gegenüber traditionellen stationären Behandlungen aufweisen kann. Soteria Bern hat auch nach über 20jährigem Bestehen Schrittmacherfunktion. Dafür sind ihr kürzlich zwei Ehrungen zuteil geworden.

\section{Ehrung für Soteria-Gründer}

Soteria Bern wurde 1984 von Luc Ciompi als therapeutische Wohngemeinschaft für die milieutherapeutische Behandlung von Psychosen gegründet. Er folgte damit dem Vorbild Loren Moshers, der in den 70er Jahren in den USA das Soteria ${ }^{1}$ Konzept als Alternative zu den «unruhigen und unpersönlichen» traditionellen Grosskliniken entwickelt hatte. Die in den US-Kliniken verabreichten Neuroleptikadosen waren exorbitant. Moshers Ziel war es in seinem Pionierprojekt, junge, akut an Schizophrenie erkrankte Menschen mittels kontinuierlicher Begleitung («being with») im reizarmen Milieu eines Wohnhauses möglichst ohne Einsatz von Neuroleptika zu behandeln. Mehrere von ihm durchgeführte Studien zeigten, dass dies möglich ist mit min- 
destens ebensoguten Ergebnissen wie in den traditionellen Kliniken. Diese Befunde geben bis heute Anlass zu kontroversen Diskussionen. Stefan Priebe hat dies folgendermassen kommentiert: «Die blosse Existenz von Soteria hat über viele Jahre die Diskussionen um die beste Form der Psychosebehandlung in kontroverser, aber insgesamt fruchtbarer Weise in Gang gehalten.»

Ciompis Soteria-Konzept unterschied sich von Anfang an in einigen wesentlichen Punkten vom radikaleren Ansatz Moshers. Für Ciompi stand eine medikamentenfreie Behandlung nicht im Zentrum. Vielmehr wollte er die in seinem 1982 erschienenen Buch «Affektlogik» formulierten Hypothesen in der alltäglichen Schizophreniebehandlung umsetzen und überprüfen. $\mathrm{Zu}$ diesem Zwecke führte er das «Weiche Zimmer» als eines der zentralen Behandlungsinstrumente der Soteria ein. Am Kongress der «International Society for the Psychotherapy of Schizophrenia (ISPS)», der im Juni 2006 in Madrid stattfand, ist Luc Ciompi mit ausdrücklichem Bezug auf das therapeutische Konzept der Soteria Bern zum «Lifetime Honorary Member» dieser Gesellschaft ernannt worden - eine hohe Ehre, die weltweit immer nur 12 Forschern zur gleichen Zeit zukommt.

\section{Das Konzept von Soteria}

Soteria Bern bietet acht Bewohnern/-innen² Platz, hat den Status eines Spitals und ist auf der Spitalliste des Kantons Bern aufgeführt. Der Träger ist die Interessengemeinschaft Sozialpsychiatrie Bern. Es werden jüngere Menschen aufgenommen, die sich in einer psychotischen Krise aus dem schizophrenen Formenkreis oder im Rahmen einer Adoleszenten- oder anderen Entwicklungskrise befinden.

Das therapeutische Milieu wird entscheidend durch eine tragende, Geborgenheit vermittelnde und reizgeschützte Atmosphäre geprägt. Die Umgebung, die Mitbewohner/innen, die Betreuer/innen und die alltäglichen Tätigkeiten im Haushalt stellen das unmittelbare therapeutische Milieu dar. Die Anforderungen des Alltags und der Gruppe stärken den Bezug zur Realität und sind Übungsfeld, um sich den Herausforderungen in der Zeit nach der akuten Krise zu stellen. Darüber hinaus ist die tätige Gemeinschaft in der Gruppe ein wichtiges Element, um sich auch wieder im Alltag zurechtzufinden.

Die Bewohner/innen werden möglichst weitgehend in den Entscheid über den Einsatz von
Medikamenten einbezogen. Ziel ist letztlich,
«Soteria Bern»:

\section{projet pionnier dans le traite- ment de la schizophrénie}

Pour nombre de cercles officiels et professionnels, le nom de Soteria est le symbole d'une approche thérapeutique en psychiatrie «plus humaine». L'offre thérapeutique se distingue par un lieu de soins extra-hospitalier, une thérapie intensive connectée au milieu, des neuroleptiques à petites doses et le recours aux proches. Les résultats de recherches effectuées aux Etats-Unis et en Suisse ont montré que l'utilisation thérapeutique à Soteria a au moins la même efficacité que la meilleure des efficacités obtenues par traitement hospitalier. Soteria Bern a prouvé son rôle de pionnier depuis plus de vingt ans. Honorant précisément son concept thérapeutique Soteria Bern, la Société internationale de psychothérapie de la schizophrénie (ISPS) a désigné Luc Ciompi en tant que «Lifetime Honorary Member».

Soteria Bern a été fondé en 1984 par Luc Ciompi en tant que communauté thérapeutique de milieu pour le traitement des psychoses. II a suivi en cela l'exemple de Loren Mosher, qui dans les années soixante-dix a développé aux Etats-Unis le concept Soteria en tant qu'alternative au modèle traditionnel «angoissant et impersonnel» des grands hôpitaux. Le but de Mosher dans son projet de l'époque consistait à traiter de jeunes patients atteints de schizophrénie aiguë au moyen d'un accompagnement continu («being with») dans un milieu dépourvu de stimuli d'excitation, comme un appartement, et le plus possible sans recours aux neuroleptiques. Le concept Soteria de Ciompi se distingue d'emblée du projet plus radical de Mosher par quelques points importants. Pour Ciompi, le traitement sans aucun médicament n'est pas central. Pour cet usage-là, un local appelé «Weiches Zimmer» est I'un des principaux éléments thérapeutiques du projet Soteria.
2 In der Soteria werden die Patienten Bewohner genannt. 


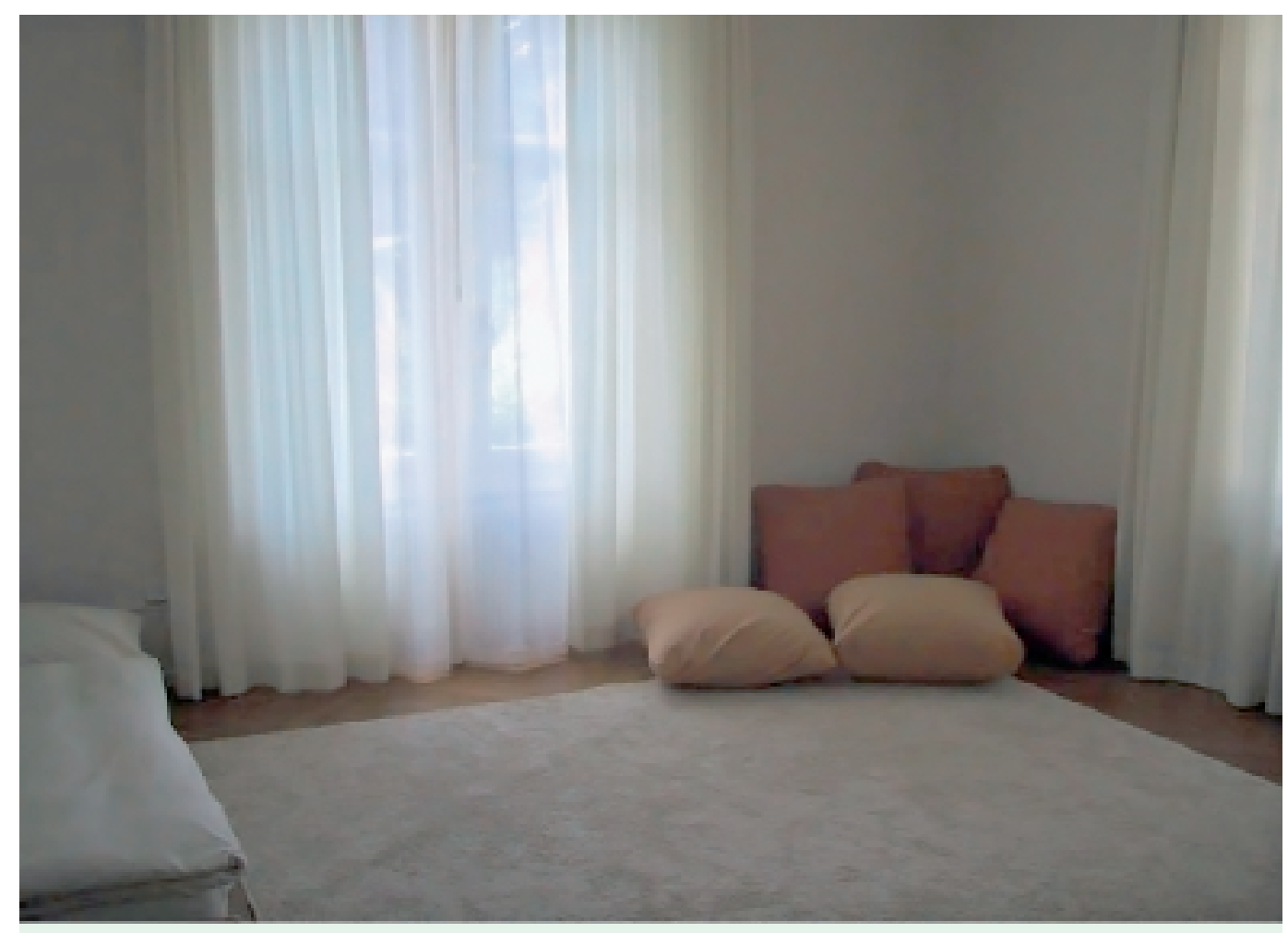

Abbildung 2

Das «Weiche Zimmer».

dass die Bewohner/innen einen auf Selbstwahrnehmung und -einschätzung basierenden und individuell angepassten Umgang mit Medikamenten lernen. Beruhigung, Reizschutz und Angstlinderung in der akuten Phase - und damit auch der Normalisierung von Denken, Fühlen und Handeln - wird in der Soteria Bern zu einem grossen Teil durch das spezifische Milieu im «Weichen Zimmer» und die tragende 1:1-Begleitung («being with») erreicht. Dank dieser besonderen Betreuung und Umgebung können Medikamente zurückhaltender als sonst üblich eingesetzt werden.

Die Bewohner/innen können mit den Betreuern/-innen Gesprächstermine vereinbaren, die aber nicht den Charakter einer Psychotherapie im engeren Sinn haben sollen. Sie haben zudem je zwei Bezugspersonen im Team, in der Regel eine Frau und einen Mann. Diese nehmen rasch nach Eintritt mit den Angehörigen Kontakt auf und laden sie zu regelmässig stattfindenden Familiengesprächen ein. Einmal pro Monat findet zudem ein Angehörigenabend statt.

Das Risiko, dass die weiterhin verletzlichen Menschen nach dem Austritt aus der Soteria Bern früher oder später wieder in schwierige Lebenssituationen oder sogar erneut in eine psychotische Krise geraten, ist erheblich. Deshalb legen wir grosses Gewicht auf die Vorbereitung des Austritts der Bewohner/innen und der Zeit nach dem Aufenthalt. Die Bezugspersonen sind für die Information über die Rückfallrisiken und eine ausführliche Besprechung vorbeugender Massnahmen verantwortlich. Die Angehörigen werden in die Rückfallprophylaxe miteinbezogen. Weil die tragende Atmosphäre der Soteria nach dem Austritt wegfällt, sind Medikamente in der Nachbehandlung als Schutz oft angezeigt.

\section{Wie wirkt Soteria? ${ }^{3}$}

Der Behandlungserfolg von Soteria basiert auf dem Zusammenwirken von psycho- und milieutherapeutischen Einzelaspekten zum Faktor der «nachhaltigen emotionalen Entspannung». Die angstlösende Umgebung wirkt vorbeugend, bessernd und auch heilend. Besonders in der akuten Psychose ist eine verständig stützende, tolerante und entspannende Atmosphäre therapeutisch wirksam. Die Sicherheit vermittelnde Grundstimmung wird mit einer klaren, der ausufernden psychotischen Verwirrung Grenzen setzenden Haltung verbunden. Grosse Wichtigkeit kommt der Reizabschirmung zu. Der sehr weitgehende Schutz vor Aussenreizen, möglichste Vertrautheit und Normalität im Umgangsstil sowie transparente Kleinräumigkeit des 
therapeutischen Milieus vermitteln emotionale Konstanz und Klarheit. Dadurch wird die psychotisch gestörte Informationsverarbeitung erleichtert. Dies ist der Grund, wieso unter Verwendung von weniger Neuroleptika der gleiche Behandlungserfolg erzielt werden kann. «Soteria wirkt neuroleptikaartig, freilich ohne die entsprechenden Nebenwirkungen» sagt Luc Ciompi treffend.

Die Alltäglichkeit der Interaktion und Kommunikation in Soteria widerspiegelt die therapeutische und tätige Gemeinschaft und verdeutlicht bisherige familiäre Muster. Verstärkt wird dies durch die Tatsache, dass die Betreuer/innen ohne Unterbruch 48 Stunden in der Soteria arbeiten und in dieser Zeit gemeinsam mit den Bewohnern/-innen den Alltag bewältigen. Ihre Funktion ist mit der von älteren Geschwistern zu vergleichen, jedoch können sie sich emotional distanzieren und abgrenzen. Soteria eröffnet durch ihre Familienähnlichkeit die Möglichkeit, kompensatorisch Prozesse nachzuholen und zu simulieren, wie sie auch in der Familie durchlaufen werden könnten.

Da emotionale Stimmungen und Haltungen bekanntlich hochgradig ansteckend sind, vermag jede Entspannung in einem Einzelsektor zudem in zirkulärem Wechselspiel die Entwicklung eines insgesamt bergenden und beruhigenden Gesamtmilieus zu fördern, in welchem Vertrauen und, auf dieser Grundlage, tiefe zwischenmenschliche Begegnungen möglich werden.

\section{Soteria im Wandel der Zeit}

Soteria Bern wurde in einer Zeit des Aufbruchs der Sozialpsychiatrie gegründet, die sich eine Verlagerung des Behandlungsschwerpunktes von der Klinik in die Gemeinde zum Ziel gesetzt hat. Diese Rahmenbedingungen haben sich seither deutlich gewandelt. Auch in den Kliniken hat Milieutherapie Einzug gefunden, die Behandlung ist patientenzentrierter geworden, die Neuroleptikadosen sind allgemein gesunken und mit den neuen atypischen Neuroleptika die Nebenwirkungen geringer. Das «Feindbild» gegenüber den Neuroleptika besteht nicht mehr.

Diesem Wandel galt es Rechnung zu tragen. Zudem haftet auch heute noch dem Namen Soteria der Mythos der Medikamentenfreiheit an und wird in allen Diskussionen zu diesem Behandlungsprinzip weiter mitgeführt. Neuroleptika wurden in Soteria Bern von Beginn an eingesetzt, anfangs noch zurückhaltender als heute. Dafür war die Aufenthaltsdauer deutlich zu lang. Auch wenn die täglichen Kosten denen der Klinik entsprachen, war Soteria ökonomisch betrachtet $\mathrm{zu}$ teuer. Zudem hatte sich Soteria von einer Wohngemeinschaft für ersterkrankte Schizophreniepatienten in Richtung einer Rehabilitationseinrichtung entwickelt. Beidem musste Gegensteuer gegeben werden.

Soteria Bern behandelt heute wieder schwerpunktmässig Akutpatienten, entsprechend ist das «Weiche Zimmer» praktisch dauernd belegt. Rückfallprophylaxe und Nachbetreuung haben in der Behandlung weiter an Bedeutung gewonnen. Die Nachfrage v.a. von seiten der Betroffenen und ihren Angehörigen ist weiter gestiegen, damit auch die Auslastung. Die Aufenthaltsdauer liegt heute mit durchschnittlich 44 Tagen unter der einer vergleichbaren Klinikstation für ersterkrankte Schizophreniepatienten. Wirtschaftlich gesehen hat Soteria Bern noch nie so erfolgreich gearbeitet wie in den letzten Jahren. Dies bestätigt auch die Erfolgskontrolle der Gesundheitsdirektion des Kantons Bern.

Soteria Bern hat also nicht starr an seinem ursprünglichen Konzept festgehalten, sondern einerseits auf die sich wandelnden Verhältnisse erfolgreich reagiert, andererseits mit innovativen Konzeptanpassungen ihre Schrittmacherfunktion immer wieder von neuem unter Beweis gestellt.

\section{Innovationen der Soteria Bern}

\section{Vermehrter Einbezug der Familie}

Obwohl der Einbezug der Angehörigen von Anfang an fester Bestandteil des Soteria-Konzeptes war, trugen die Angehörigengruppe und die gelegentlichen Familiengespräche den Therapiezielen der Soteria zu wenig Rechnung. Wir intensivierten und professionalisierten deshalb die Familiengespräche in Richtung Familientherapie und boten für die Mitarbeiter/innen regelmässige Supervision an. Damit gelang es uns, die Angehörigen zu entlasten, die emotionale Spannung innerhalb der Familie zu senken und gemeinsam Lösungswege im Umgang mit dem psychotischen Geschehen zu finden.

\section{Ehemaligengruppe}

Die Bewohner/innen werden eingeladen, sich nach dem Austritt - sei es in einer Krise oder wenn es ihnen danach ist - in der Soteria zu melden. Von diesem Angebot wurde all die Jahre rege Gebrauch gemacht. Dies ermutigte uns, 2004 mit einer Gruppe für Ehemalige zu starten, die sich zunehmender Beliebtheit erfreut.

\section{Cannabis und Psychose}

Bei der Behandlung junger, akut an Schizophrenie Erkrankter konnten wir in den letzten Jahren 
in der Soteria Bern eine ständige Zunahme von Patienten beobachten, die z.T. sehr intensiv und/oder langdauernd Cannabis konsumieren. So waren es $200563 \%$ aller Eintritte $(n=60)$ oder $76 \%$ der unter Dreissigjährigen.

Aus mehreren Studien wissen wir heute, dass Cannabiskonsum zu einem früheren Ausbruch der Schizophrenie führt, v.a. wenn dieser bereits sehr früh (<15 Jahre) beginnt, intensiv ist und bereits einmal zu einem psychotischen Erleben nach Cannabiskonsum geführt hat.

Wir sind deshalb bereits 2004 dazu übergegangen, während des Aufenthaltes in der Soteria eine Abstinenz vom Cannabis von den Bewohner/innen zu fordern und sie im Sinne der Rückfallprophylaxe zu einem dauerhaften Verzicht auf Cannabis zu motivieren. In der Literatur fanden wir keine Therapieprogramme, die uns in unseren Anstrengungen hätten unterstützen können. Wir beschlossen deshalb, ein solches für die Soteria zu entwickeln. Dies soll eine Erweiterung des Therapieangebots der Soteria Bern darstellen, das den Bewohnern/-innen helfen soll, auch über den Aufenthalt hinaus nachhaltig auf den Konsum von Cannabis zu verzichten. Für dieses Angebot wurde uns an der diesjährigen Jahrestagung der Schweizerischen Gesellschaft für Psychiatrie und Psychotherapie der Prix Perspective verliehen.

\section{Integrierte Versorgung}

Gemäss dem Konzept der Soteria Bern hat die Rückfallprophylaxe einen hohen Stellenwert, weshalb die Soteria Bern als Erweiterung ihres Angebotes Übergangswohngemeinschaften mit Soteria-Elementen für jeweils 2-3 Bewohner/innen unter dem Label «Wohnen \& Co.» anbieten möchte. Mit diesem Angebot kann nach dem in den letzten Jahren kürzer gewordenen Aufenthalt in der Soteria Bern die Behandlungskontinuität und damit die Stabilisierung über den stationären Aufenthalt hinaus erhöht und das Risiko einer erneuten Hospitalisation oder eines Rückfalles in den Cannabiskonsum vermindert werden. Damit liesse sich die Nachhaltigkeit der Soteria-Behandlung wesentlich verbessern. Dies ist konzeptuell ein wichtiger Schritt in Richtung integrierter Versorgung mittels fliessendem Übergang von stationärer, komplementärer hin zur ambulanten Betreuung, verbunden mit der Behandlungskontinuität durch den Case Manager.

\section{Ausstrahlung}

Im deutschen Sprachraum ist das Interesse für den Soteria-Ansatz in den letzten Jahren sehr stark gestiegen. So sind in der Zwischenzeit drei weitere Soterias in Frankfurt/Oder, Zwiefalten und München-Haar entstanden. Erstere musste wegen mangelnder Sicherstellung der Finanzierung wieder geschlossen werden. Zudem sind mehrere «Stationen mit Soteria-Elementen» entstanden. Daneben gibt es mehrere Projektgruppen (auch wieder in den USA), meist erst auf der Planungsebene, vereinzelt dagegen auch schon auf der Ebene der praktischen Verwirklichung. Erheblich gewachsen ist ebenfalls das einschlägige Schrifttum, wobei aber Veröffentlichungen mit wissenschaftlich evaluativem Charakter immer noch deutlich in der Minderzahl sind. 1997 konnten wir in Bern die «Internationale Arbeitsgemeinschaft Soteria» gründen, deren jährliche Treffen immer mehr Teilnehmer anzieht.

Die Soteria-Idee ist keineswegs nur ein romantisches Relikt aus der angestrebten «menschlicheren» Psychiatrie bzw. «Antipsychiatrie» der Sechziger- und Siebzigerjahre, sondern vielmehr (vermutlich in abgewandelter, aber nicht ihres Kerns entfremdeter Form) die (oder doch eine) gute Lösung für eine Psychosebehandlung der Zukunft. Denn wenn tatsächlich, wie es jetzt immer klarer wird, die Umwelt- und subjektive Beziehungssituation einen tiefen Einfluss auf die ganze Hirnfunktion hat, so bietet kein anderes uns bekanntes Milieu als das der Soteria optimale Bedingungen, um eine psychotisch verstörte Hirnfunktion zu verbessern. Therapeutische Wohngemeinschaften vom Typ der Soteria dürfen deshalb als Hoffnungsträger und attraktives Schrittmachermodell für die Schizophreniebehandlung des 21. Jahrhunderts gesehen werden.

\section{Literatur}

- Ciompi L, Hoffmann H, Broccard M (Hrsg.). Wie wirkt Soteria? Eine atypische Psychosenbehandlung kritisch durchleuchtet. Bern, Göttingen, Toronto, Seattle: Verlag Hans Huber; 2001.

- Ciompi L, Hoffmann H. Soteria Berne - an innovative milieu therapeutic approach to acute schizophrenia based on the concept of affect-logic. World Psychiatry. 2004;3:140-6. 